\title{
Assessment of a Non Invasive Brain Oximeter in Volunteers Undergoing Acute Hypoxia
}

This article was published in the following Dove Press journal:

Medical Devices: Evidence and Research

\author{
Barry Dixon (1D) \\ David B MacLeod (1D) ${ }^{2}$ \\ 'Cyban Pty Ltd, Melbourne, VIC, \\ Australia; ${ }^{2}$ Human Pharmacology and \\ Physiology Laboratory, Department of \\ Anesthesiology and School of Nursing, \\ Duke University, Durham, NC, USA
}

Correspondence: Barry Dixon Tel +6I 04396I88I5

Email barry.dixon@cyban.com.au
Introduction: Research in traumatic brain injury suggests better patient outcomes when invasive oxygen monitoring is used to detect and correct episodes of brain hypoxia. Invasive brain oxygen monitoring is, however, not routinely used due to the risks, costs and technical challengers. We are developing a non-invasive brain oximeter to address these limitations. The monitor uses the principles of pulse oximetry to record a brain photoplethysmographic waveform and oxygen saturations. We undertook a study in volunteers to assess the new monitor.

Patients and Methods: We compared the temporal changes in the brain and skin oxygen saturations in six volunteers undergoing progressive hypoxia to reach arterial saturations of $70 \%$. This approach provides a method to discriminate potential contamination of the brain signal by skin oxygen levels, as the responses in brain and skin oxygen saturations are distinct due to the auto-regulation of cerebral blood flow to compensate for hypoxia. Conventional pulse oximetry was used to assess skin oxygen levels. Blood was also collected from the internal jugular vein and correlated with the brain oximeter oxygen levels.

Results: At baseline, a photoplethysmographic waveform consistent with that expected from the brain was obtained in five subjects. The signal was adequate to assess oxygen saturations in three subjects. During hypoxia, the brain's oximeter oxygen saturation fell to $74 \%$, while skin saturation fell to $50 \%(\mathrm{P}<0.0001)$. The brain photoplethysmographic waveform developed a high-frequency oscillation of $\sim 7 \mathrm{~Hz}$, which was not present in the skin during hypoxia. A weak correlation between the brain oximeter and proximal internal jugular vein oxygen levels was demonstrated, $\mathrm{R}^{2}=0.24, P=0.01$.

Conclusion: Brain oximeter oxygen saturations were relatively well preserved compared to the skin during hypoxia. These findings are consistent with the expected physiological responses and suggest skin oxygen levels did not markedly contaminate the brain oximeter signal.

Keywords: oximetry, brain injury, brain, volunteer, hypoxia, monitor

\section{Plain Language Summary}

Following severe traumatic brain injury episodes of brain hypoxia commonly develop. No simple and safe non invasive method to monitor oxygen levels in the brain and guidetreatments is currently available. Brain hypoxia may therefore be recognised late, afterirreversible brain injury has occurred.

We are developing a non invasive method to continuously monitor brain oxygen saturations. The monitor was assessed in 6 human volunteers undergoing hypoxia. We found the baseline brain PPG waveform was different from the simultaneous skin arterial PPG waveform. The brain PPG waveform, was similar in shape and timing to a central venous pressure trace, with A, X, V and Y waves. Brain oxygen saturations also fell markedly during diastole. The brain PPG waveform but not the skin PPG, developed distinctive changes during periods of hypoxia. In addition, the brain oximeter oxygen saturations were relatively well preserved 
compared with the skin during hypoxia. These findings are consistent with the expected physiological responses and suggest skin oxygen levels did not markedly contaminate the brain signal.

\section{Introduction}

Following severe traumatic brain injury (TBI), 55\% of patients develop a secondary brain injury in the minutes, hours, days and weeks after the initial insult. The complications giving rise to hypoxia include brain swelling, hydrocephalus, vasospasm and low blood pressure. ${ }^{1,2}$ Opinion is emerging that treatments guided by brain oxygen monitoring to detect and minimise episodes of brain hypoxia reduces mortality and long-term disability., ${ }^{1,3-5}$

Brain monitoring following trauma, however, continues to be problematic. Available options are all invasive, including intra-cranial pressure (ICP) catheters, intra-parenchymal oxygen probes and jugular bulb venous lines. These approaches can result in adverse events, are technically challenging, expensive, yet may not detect all episodes of hypoxia, and only provides monitoring for a relatively short period, that does not include ambulance retrieval or in hospital locations other than critical care units., ${ }^{2,4,6}$ Due to these limitations, only $10 \%$ of patients with severe TBI currently have brain oxygen levels monitored. ${ }^{7,8}$ Other, non-invasive monitors, such as cerebral oximeters, are inaccurate in the setting of acute brain injury and are not used to guide treatment. $^{9,10}$ This is believed to result largely from skin contamination of their signal. Expert guidelines therefore do not recommend their use. ${ }^{11-13}$

We are developing, a new brain oximeter based on the principles of pulse oximetry, designed to detect the brain photoplethysmographic waveform and measure brain oxygen saturations to address the limitations of invasive brain monitoring. Assessment of the oximeter in a sheep model of acute brain injury demonstrated it identified a brain photoplethysmographic waveform and acute changes in brain oxygen saturations associated with increases in ICP levels. ${ }^{14}$

This study assesses the brain oximeter in human volunteers undergoing an acute hypoxia challenge. We compared the temporal changes in the brain and skin oxygen saturations, as brain oxygen levels are preserved relative to the skin during hypoxia due to a compensatory increase in cerebral blood flow to maintain brain oxygen levels. ${ }^{15,16}$ This approach provides a method to discriminate potential contamination of the brain signal by oxygen levels in the skin, which is a limitation of existing cerebral oximeters. ${ }^{17,18}$
The aims of the study were to assess the temporal changes in brain and skin oxygen saturations during acute hypoxia and to measure the correlation between the brain oximeter and proximal internal jugular vein oxygen saturations.

\section{Patients and Methods}

The study assessed a new oximeter at a number of internal organ sites including the brain. This paper reports on the results for the brain only. The study was approved by the Duke University Institutional Review Board. The study was conducted in accordance with the Declaration of Helsinki. Six healthy volunteers who gave written informed consent were enrolled. Three were female, the average age was 29 years, weight $72 \mathrm{~kg}$, height $172 \mathrm{~cm}$ and Body Mass Index 24 . Four volunteers had darkly pigmented skin. Volunteers underwent an extensive pre-study assessment including medical history, physical examination, blood and urine tests, electrocardiography (ECG), spirometry and, for female subjects, a pregnancy test. Enrolled subjects were fasted overnight; no sedation or anesthesia was administered.

The brain oximeter utilizes light-emitting diodes with wavelengths in the near infra-red (NIR) range 660 and 895 $\mathrm{nm}$, with a PIN photodetector. The optical power is similar to that used in conventional skin pulse oximetry. The brain oximeter uses the principles of pulse oximetry to detect a brain photoplethysmographic (PPG) waveform. ${ }^{19}$ The sensor was placed on the right forehead. Figure 1. This position

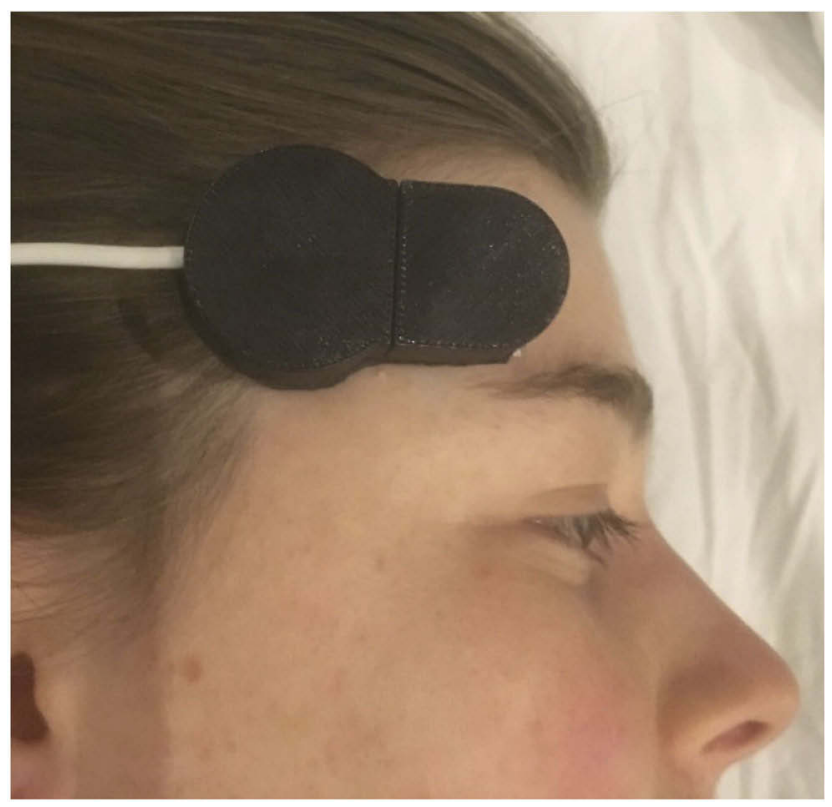

Figure I The brain oximeter sensor on the right forehead of a subject. 
was maintained with an elasticized head band. To address potential contamination of the brain signal by extra-cranial sources, the brain oximeter has a number of new design elements. Pulse oximeters determine the blood oxygen saturation by deriving the ratio between red and infrared plethysmograms. This is termed the Ratio of Ratios (RR). In conventional pulse oximetry, the displayed $\mathrm{SpO}_{2}$ value is derived from sampling the RR at the peak of the systolic pulse and represents the arterial oxygen level. The brain oximeter PPG waveform differs from the conventional skin PPG waveform. Consequently, a novel algorithm was created that ascertains a modified $R R\left(R_{m}\right)$. The brain oximeter measures microvascular blood oxygen saturations continuously throughout the cardiac cycle (systole and diastole), at a rate of $500 \mathrm{~Hz}$. This is quite distinct from skin pulse oximetry which measures the arterial blood oxygen saturation at the peak of the pulse only and also distinct from cerebral oximeters which measures the average oxygen saturation across the various blood compartments of the brain (arterial, microcirculation and venous).

To ensure patient safety and provide additional certainty around changes in arterial oxygen levels, additional monitors were applied including 5-lead ECG, forehead Max Fast pulse oximeter, 2 finger pulse oximeters, and Nexfin finger non-invasive, continuous cardiac output and blood pressure monitor (BMEYE BV., Amsterdam, Netherlands). The right internal jugular vein was cannulated and the tip of a 14 Gauge cannula located was at the proximal end of the vein using ultrasound guidance. The monitor's sensor was placed on the right forehead. Both an anesthesiologist and a critical care physician were present to oversee study procedures, volunteer safety and data validity.

\section{Hypoxia}

A facemask and breathing apparatus with a tight seal (ROBD2, Environics Inc, Tolland, CT) were used to induce controlled hypoxia. The ROBD2 devices deliver a specific inspired concentration of oxygen fraction $\left(\mathrm{F}_{\mathrm{i}} \mathrm{O}_{2}\right)$ which is then reduced in small increments to attain the target pulse oximeter oxygen saturation, range of $100 \%$ to $10 \%$. The desaturation protocol consisted of 9 sequential stages: Room air followed by hypoxic gas mixture to attain arterial $\mathrm{SpO} 2$ values of $94 \%, 90 \%, 85 \%, 80 \%, 75 \%$ and $70 \%$, then $100 \%$ oxygen and finally room air. Once a stable plateau $\mathrm{SpO} 2$ saturation was reached (typically within 3-5 mins), 4 jugular venous blood samples were collected over approximately 4-5 mins and the venous oxygen saturation immediately measured with a co-oximeter (ABL 90, Radiometer, Copenhagen, Denmark) to measure jugular venous oxygen saturations. At completion of the study, all catheters were removed, and insertion sites inspected for hematoma; all subjects were contacted by telephone the following day to verify health status.

\section{Brain and Skin Oximetry}

Oxygen levels were averaged over 5 second windows to allow the temporal changes with hypoxia to be pooled for all participants. As brain microvascular oxygen saturations vary markedly during the systolic and diastolic phases of the cardiac cycle, the data presented represent the average oxygen saturation over the whole cardiac cycle. To determine brain microvascular blood oxygen saturations, as the exact correlation of $\mathrm{RR}_{\mathrm{m}}$ with blood oxygen saturations is yet to be established, we used the formula $\mathrm{SO}_{2}=$ $110-25\left(\mathrm{RR}_{\mathrm{m}}\right)$ to estimate the oxygen saturation $\left(\mathrm{SO}_{2}\right){ }^{19}$ The same method was used to estimate skin oxygen saturation, to enable valid comparison oxygen levels at the 2 sites. The figures demonstrating light intensity changes from the skin and brain pulse oximeters are presented, with the $\mathrm{Y}$ axis (light intensity) inverted, by convention.

\section{Correlation Analysis}

For each stage of the desaturation, the 4 blood gases sampled were averaged and correlated with the average of the $R_{\mathrm{m}}$ measured by the brain oximeter over the same period.

\section{Statistical Analysis}

Least squares linear regression assessed the relationship between jugular venous oxygen saturation with the brain oximeter, unpaired $t$-test assessed the change in volunteers cardiovascular responses to hypoxia and repeated measures analysis were undertaken to assess the temporal responses between skin and brain oxygen saturations to acute hypoxia, using Prism software (Version 8, Graph Pad Inc, San Diego, USA).

\section{Results}

Six volunteers were studied. Blood pressure, heart rate and cardiac index, all increased significantly with hypoxia (Table 1).

A PPG waveform consistent with the expected brain waveform was obtained in 5 patients. In 2 of these 5 subjects, the 
Table I Cardiovascular Responses to Hypoxia. The Baseline and Maximum Levels (During Hypoxia) are Shown

\begin{tabular}{|l|l|l|l|}
\hline Characteristics & $\begin{array}{l}\text { Baseline } \\
(\mathbf{N}=6)\end{array}$ & $\begin{array}{l}\text { Hypoxia } \\
(\mathbf{N}=6)\end{array}$ & P value \\
\hline Heart rate (beats/min) & $52 \pm 13$ & $83 \pm 15$ & 0.002 \\
$\begin{array}{l}\text { Mean blood pressure } \\
(\mathrm{mmHg})\end{array}$ & $75 \pm 6$ & $87 \pm 7$ & 0.005 \\
Cardiac index $\left(\mathrm{L} / \mathrm{min} / \mathrm{m}^{2}\right)$ & $3.0 \pm 0.6$ & $4.6 \pm 0.9$ & 0.006 \\
\hline
\end{tabular}

signal-to-noise ratio was too low to allow the algorithm to determine oxygen saturations. The oxygen data presented therefore represent only 3 of the 6 subjects studied. The skin pulse oximetry data was from the right index finger in 2 subjects and a forehead skin pulse oximeter in 1 subject.

The brain PPG waveform at baseline is demonstrated in one subject in Figure 2. It is distinctly different from the simultaneous skin PPG waveform, which is similar in shape and timing to an arterial pressure trace. The brain pulse waveform, is similar in shape and timing to a central venous pressure trace, with $\mathrm{A}, \mathrm{X}, \mathrm{V}$ and $\mathrm{Y}$ waves. ${ }^{20}$ In addition, the start of the PPG waveform was delayed relative to the start of the simultaneous forehead skin PPG waveform and the peak was at the end of diastolic phase of the cardiac cycle, rather than in systole.

Baseline oxygen saturations in the forehead skin remained relatively constant at 100-90\%, throughout the cardiac cycle. The brain oximeter oxygen saturations based on the $R_{m}$ levels were, however, not constant during the cardiac cycle. The oxygen saturations were highest during the systolic phase at $100 \%$ and thereafter levels fell markedly to $\sim 60 \%$, at the end of diastole, as shown in Figure 3.

\section{Brain and Skin Oxygen Responses to Acute Hypoxia}

At baseline, in the pooled data from the 3 subjects analysed, the oxygen saturations (averaged over systole and diastole) derived from the $\mathrm{RR}_{\mathrm{m}}$ levels were similar for both brain and skin $86 \%$ vs $87 \%$, respectively. At the

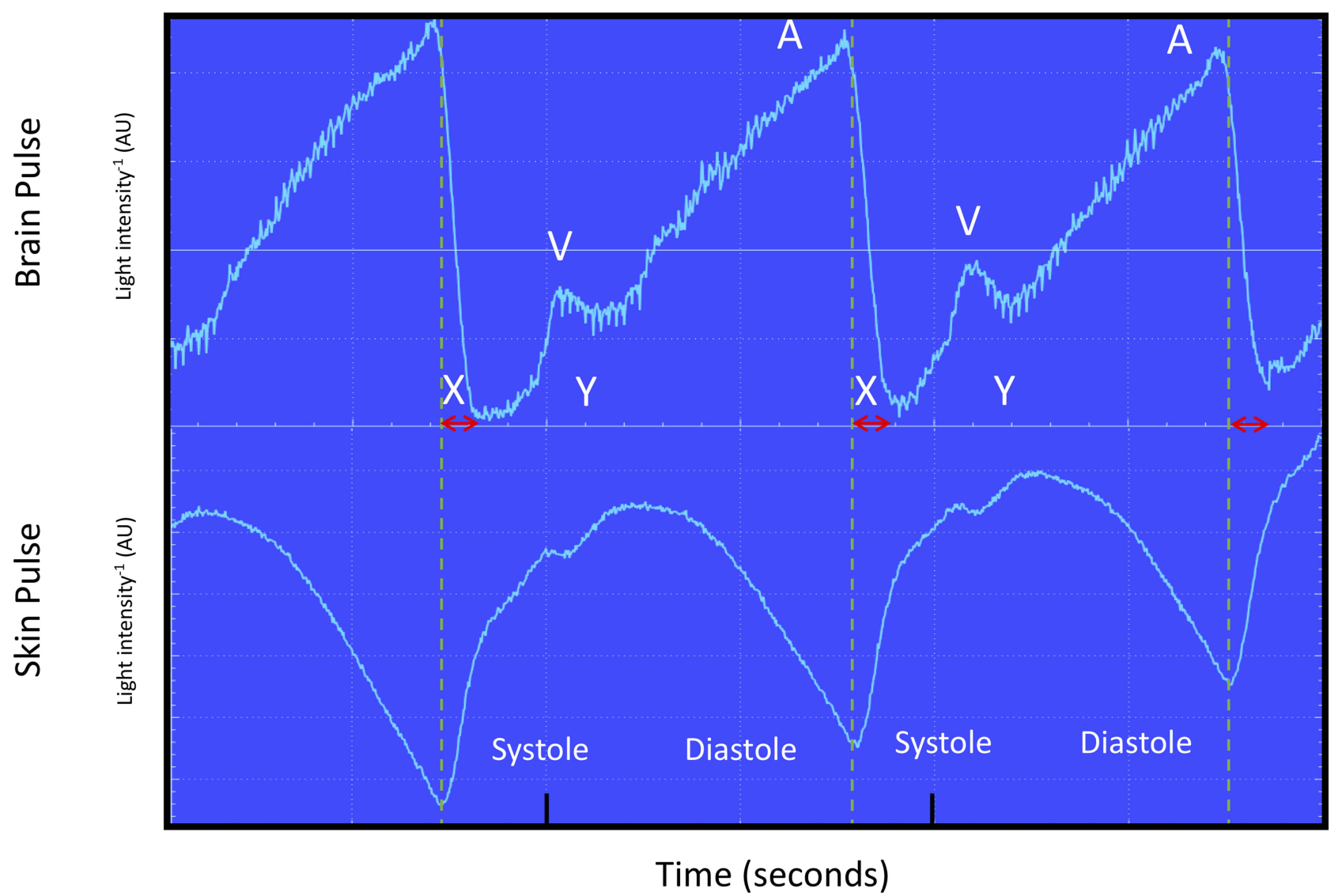

Figure 2 Recording of simultaneous brain oximeter and conventional forehead PPG waveforms ( 660 nm wavelength shown) during normoxia from subject 3 . The dashed lines represent the start of each skin pulse. The brain and forehead skin PPG waveforms were different. The brain oximeter PPG waveform was similar in shape and timing to a central venous pressure waveform, with phases consistent with $A, X, V$ and $Y$ waves. The skin PPG waveform was quite distinct with the shape and timing to an arterial pressure waveform. The brain oximeter pulse was delayed relative to the skin pulse, by $\sim 100 \mathrm{msec}$ (arrow) and the peak level was reached during late diastole. 


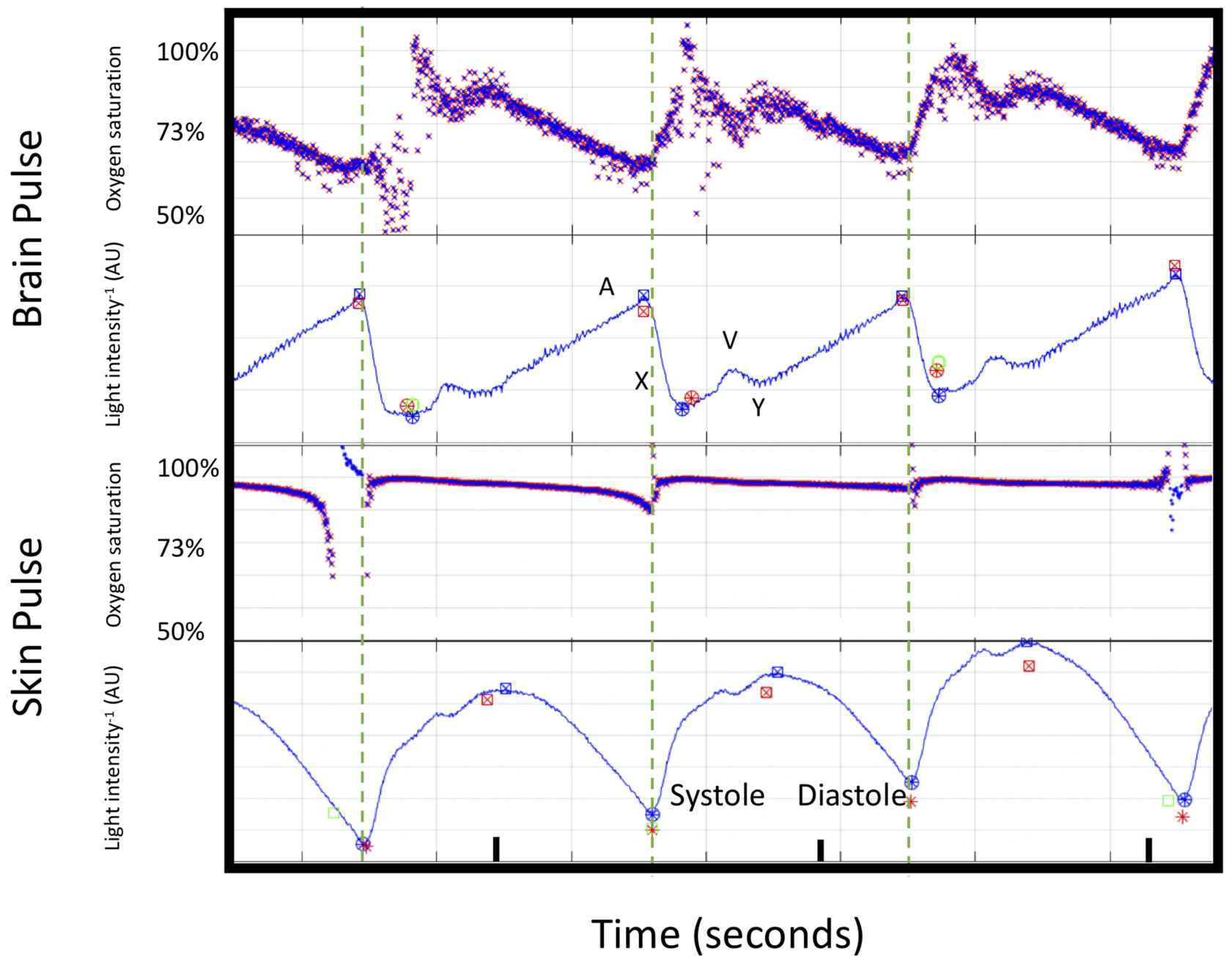

Figure 3 Cardiac cycle changes in oxygen saturations and PPG waveforms ( $660 \mathrm{~nm}$ wavelength shown) for the brain and forehead skin pulse oximeter during normoxia from subject 3. The blood oxygen saturations are demonstrated on the Yaxis. The dashed line demonstrates the start of each skin PPG waveform. The brain oximeter oxygen saturations peaked during systole, with a second lower peak during early diastole. Thereafter, oxygen saturations fell. The skin oxygen saturations remained stable throughout the cardiac cycle. The large changes in the brain oximeter oxygen saturations are consistent with the signal arising from microcirculation blood. The changes in the skin are consistent with shunting, and blood bypassing the capillaries.

Abbreviation: AU, arbitrary units.

Inspired $\mathrm{O}_{2}$

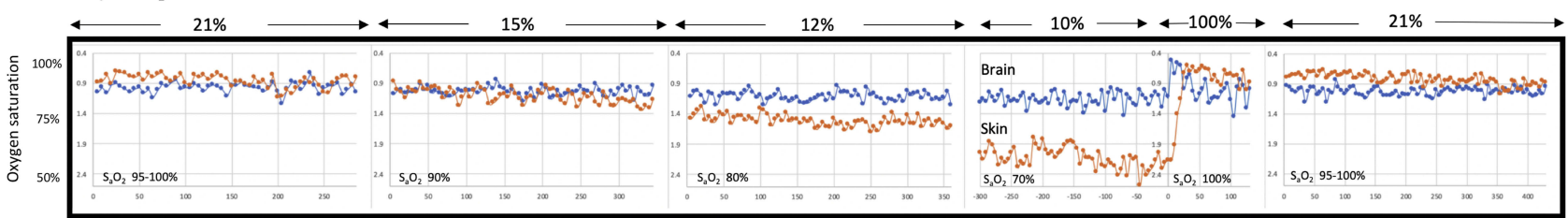

Time (seconds)

Figure 4 Brain (blue) and Skin (orange) pulse oximeter oxygen saturation levels and modified ratio of ratios (within figure Yaxes) following reductions in inspired oxygen $\left(\mathrm{O}_{2}\right)$ levels. The approximate inspired $\mathrm{O}_{2}$ are shown at the top of each panel and the targeted arterial oxygen saturation $\left(\mathrm{S}_{\mathrm{a}} \mathrm{O}_{2}\right)$ at the bottom of each panel. Brain oxygen saturations fell $1 / 3$, compared with the fall in skin oxygen saturations, at the point of maximum hypoxia $(P<0.000 \mathrm{I}$, repeat measures analysis for group). The oxygen saturation represents the average over the entire cardiac cycle. Pooled data for 3 subjects, (volunteers I, 2 and 6 ). 
point of minimum oxygen levels (arterial saturation $\sim 70 \%$ ), brain oxygen saturations fell to $74 \%$, while skin saturations fell to $50 \%$ [P $<0.0001$, repeat measures analysis, for the effect of the group]. Figure 4.

\section{Brain and Skin Oxygen Responses with Change from 10-100\% Inspired Oxygen}

The temporal responses of brain and skin oxygen saturations, in the pooled data from 3 subjects analysed, were also different in response to the increase in inspired oxygen from $10 \%$ to $100 \%$. Acute brain hyperoxia developed in the first 5 seconds following this change reaching a saturation of $99 \%$, thereafter oxygen saturations fell. Skin oxygen saturations responded much more slowly and peaked after 25 seconds. Figure 5 .

\section{Brain PPG Waveform with Hypoxia}

During periods of hypoxia the brain oximeter PPG waveform developed episodes of regular high-frequency oscillations, at around $7 \mathrm{~Hz}$, in all 3 subjects. These oscillations were synchronized with the cardiac pulse. These changes were not present in the skin PPG waveform. Figure 6.

In addition, the brain PPG waveform demonstrated an earlier and steeper rise during the systolic phase and the $\mathrm{V}$ and $\mathrm{Y}$ waves were less prominent, compared with the baseline PPG waveform. These changes were not present in the skin PPG waveform, monitored synchronously. Figure 7.

\section{Correlation with Internal Jugular Venous Blood Oxygen Levels}

A weak correlation between the monitors blood oxygen saturations and the invasive internal jugular vein blood oxygen saturations was demonstrated, in the 3 subjects. $\mathrm{R}^{2}=$ $0.24, P=0.01$. The correlation was improved with post hoc censoring of values, in which the brain PPG waveform developed a much steeper rise during the systolic phase of the waveform, $\mathrm{R}^{2}=0.63$ and $P<0.0001$, as shown in Figure 8 .

\section{Discussion}

We found in human volunteers undergoing acute hypoxia brain oximeter oxygen saturations were relatively well preserved compared with the skin, falling by $1 / 3$ of the fall in skin oxygen levels. These findings are consistent with the expected physiological responses in the brain and skin to hypoxia. They also suggest that skin oxygen levels did not markedly contaminate the signal, as is an issue with conventional cerebral oximeters. ${ }^{17,18}$

Other findings from this study, including the distinctive brain PPG waveform shape and timing relative to the skin PPG waveform, the large changes in brain oxygen saturations during systole and diastole and the $7-\mathrm{Hz}$ oscillation in the brain PPG waveform during periods of hypoxia. These findings were consistent with the signal arising from the brain and were analogous with the findings from our earlier study in a sheep model of acute brain injury. ${ }^{14}$

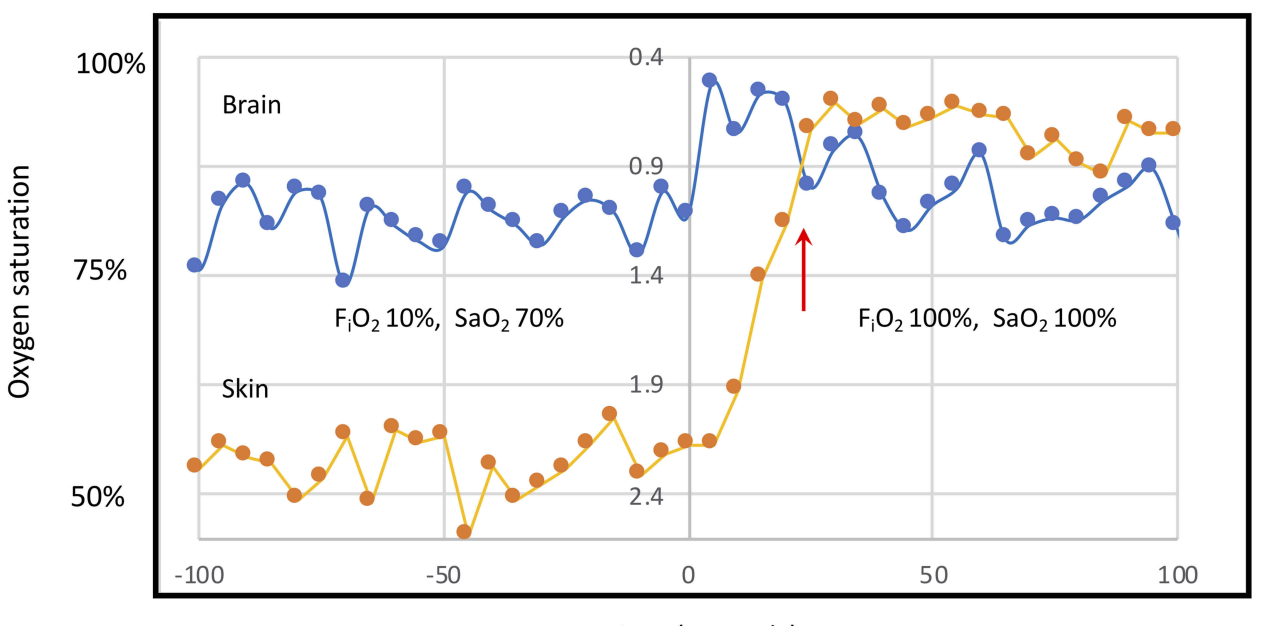

Time (seconds)

Figure 5 Brain (blue) and Skin (orange) pulse oximeter oxygen saturation levels and modified ratio of ratios (within figure $Y$ axes) following the increase in the inspired oxygen levels $\left(\mathrm{F}_{\mathrm{i}} \mathrm{O}_{2}\right)$ from $\sim 10 \%$ to $100 \%$ at time 0 . The targeted arterial oxygen saturations $\left(\mathrm{S}_{\mathrm{a}} \mathrm{O}_{2}\right)$ are shown on the figure. Brain oximeter oxygen saturations peaked within 5 seconds of the increase in $\mathrm{F}_{\mathrm{i}} \mathrm{O}_{2}$, quickly reaching hyperoxic levels and fell thereafter, while skin oxygen levels continued to increase until 25 seconds. The red arrow demonstrates a point at which the direction of change in brain and skin oximeter saturations were opposite. These distinct responses may represent the tight autoregulation of cerebral blood flow to maintain stable brain oxygen saturations. These findings suggest the brain oximeter is not significantly influenced by skin oxygen levels. The oxygen saturation represents the average level over the entire cardiac cycle. Pooled data of 3 subjects, (volunteers I, 2 and 6). 


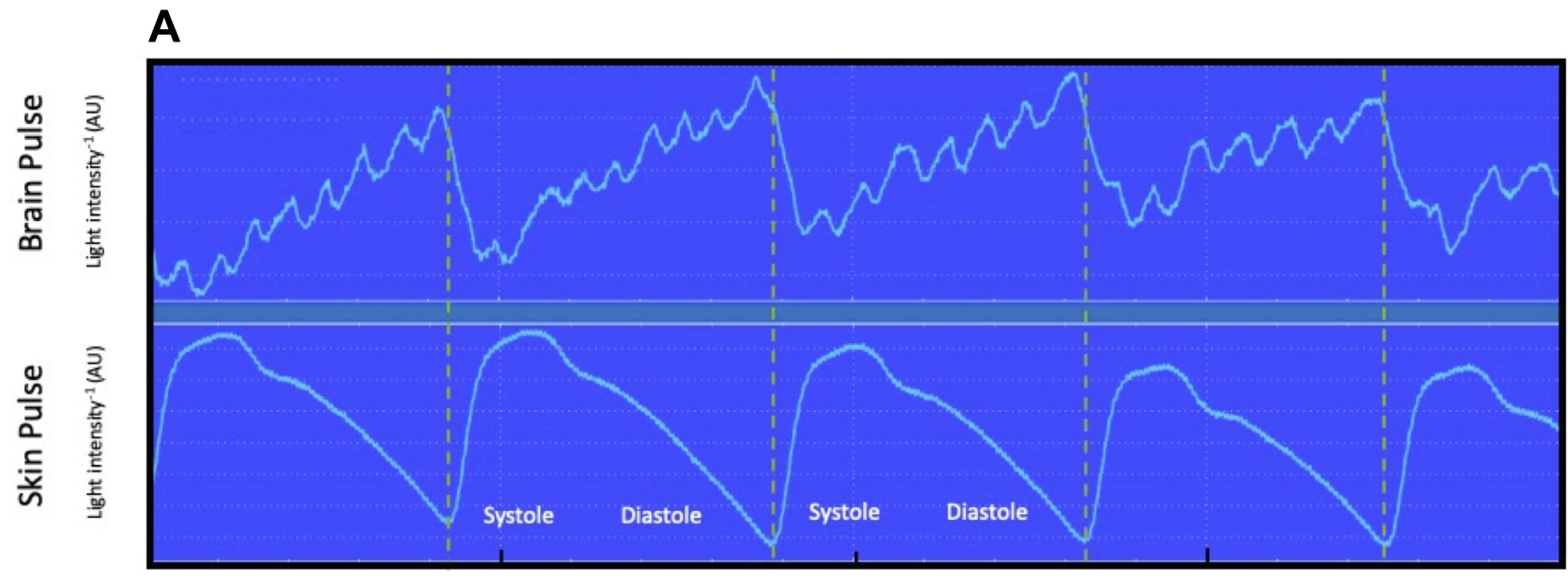

Time (seconds)

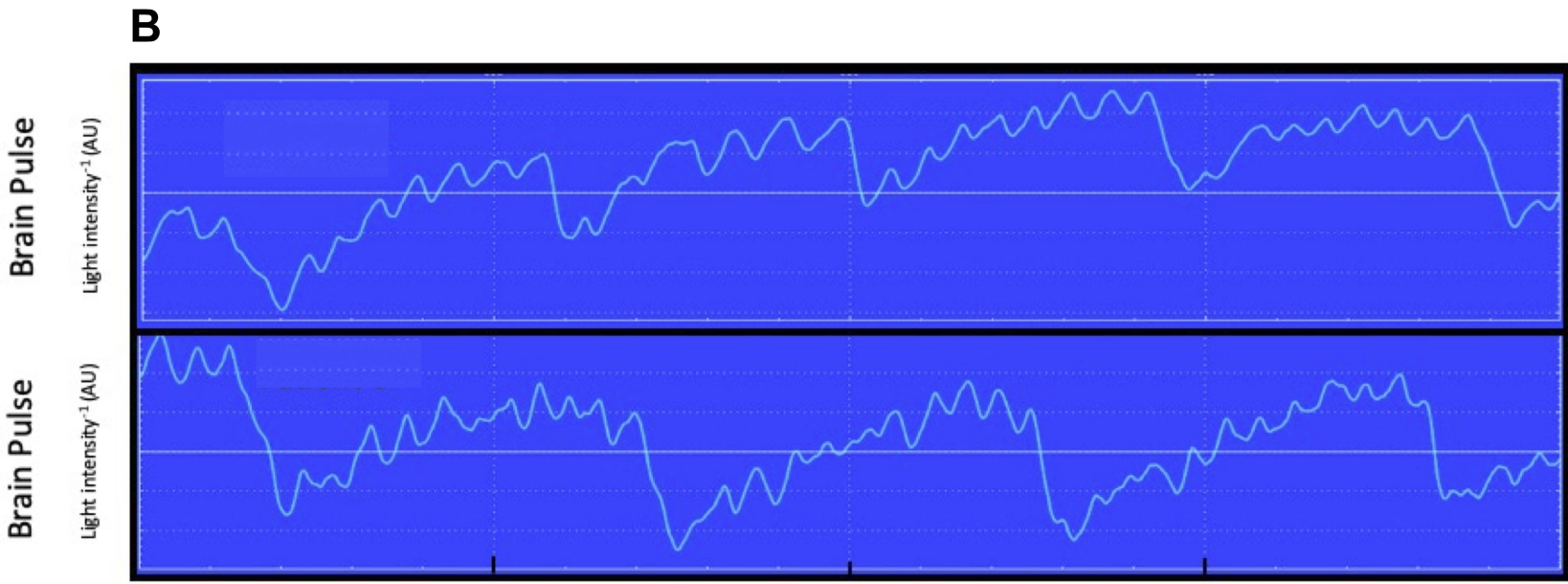

Time (seconds)

Figure 6 (A) Brain and skin oximeter simultaneous recording of PPG waveforms during hypoxia (inspired oxygen I5\%), for subject I. A high-frequency oscillation ( 7 Hz) was present in brain PPG waveform, but not the skin PPG waveform. The dashed lines represent the start of each skin pulse. Oscillations at this frequency in physiological recordings have been demonstrated for intra-cranial pressure in humans and represent physical movement "ringing" of the brain in response to the systolic pressure wave entering the brain substrate. (B) Shows similar high-frequency oscillations in subjects 2 and 6 .

Abbreviation: AU, arbitrary units.

\section{Brain PPG Waveform and Oxygen Saturations}

The PPG waveform of skin pulse oximetry is similar in shape and timing to an arterial pressure waveform, with a rise in systole and fall in diastole. The brain oximeter PPG waveform was, however, quite different with a shape and timing akin to a central venous pressure waveform, including phases consistent with $\mathrm{A}, \mathrm{X}, \mathrm{V}$ and $\mathrm{Y}$ waves. This may reflect the cardiac cycle that causes pressure variations in the central venous circulation and therefore the brain microcirculation. ${ }^{20}$ The brain PPG waveforms start was also delayed relative to the simultaneous skin PPG waveform by $\sim 100 \mathrm{msec}$ and the peak of the pulse occurred late in diastole. These characteristics suggest that the brain PPG reflected changes of blood volume in the venous circulation. This is consistent with the known large percentage of cerebral blood in the venous circulation. Proportionally the venular beds account for $50 \%$ of the brain microvascular blood and up to $85 \%$ if small veins are included. ${ }^{21-25}$ By contrast, there is extensive shunting of blood in the skin, bypassing capillary beds and venules, which gives rise to the arterial shape of skin pulse oximetry. ${ }^{26}$

The change in oxygen levels during the systolic and diastolic phases of the cardiac cycle was also distinct from that of the skin. The brain oximeter oxygen saturation was $100 \%$ during systole and fell markedly at the end of diastole to $60 \%$. The overall findings, in terms of the central venous 


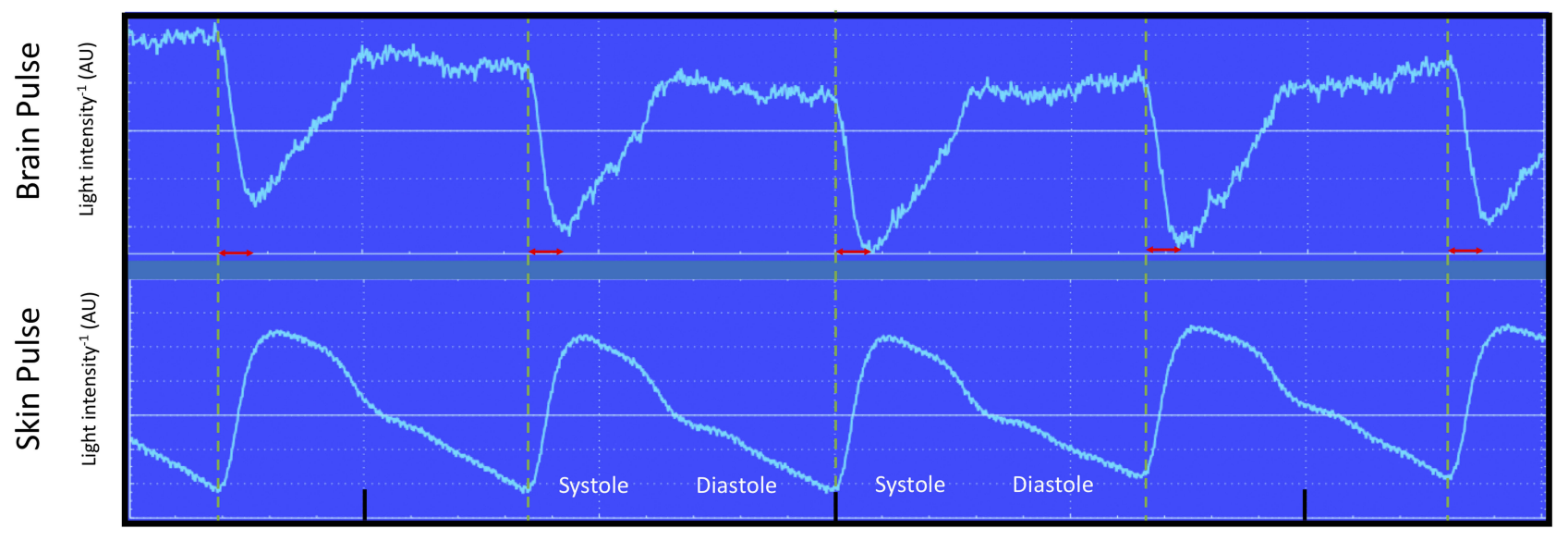

Time (seconds)

Figure 7 Simultaneous recording of brain and forehead skin PPG waveforms during a period of hypoxia (inspired oxygen 10\%), for a single subject (volunteer 6). Dashed lines mark the start of each skin pulse. The brain oximeter PPG waveform developed an earlier and steeper rise during the systolic phase and the $V$ and $Y$ waves were less prominent, compared with the baseline PPG waveform (see Figure I). These changes were not present in the simultaneous skin PPG waveform. These changes may reflect an increase in cerebral blood flow during hypoxia, resulting in a more rapid increase of blood volume in the brain microcirculation and therefore an earlier upstroke in the brain PPG waveform. Note the start of the brain PPG waveform is delayed by around 100 msec compared to the skin (red arrows).

Abbreviation: $\mathrm{AU}$, arbitrary units.

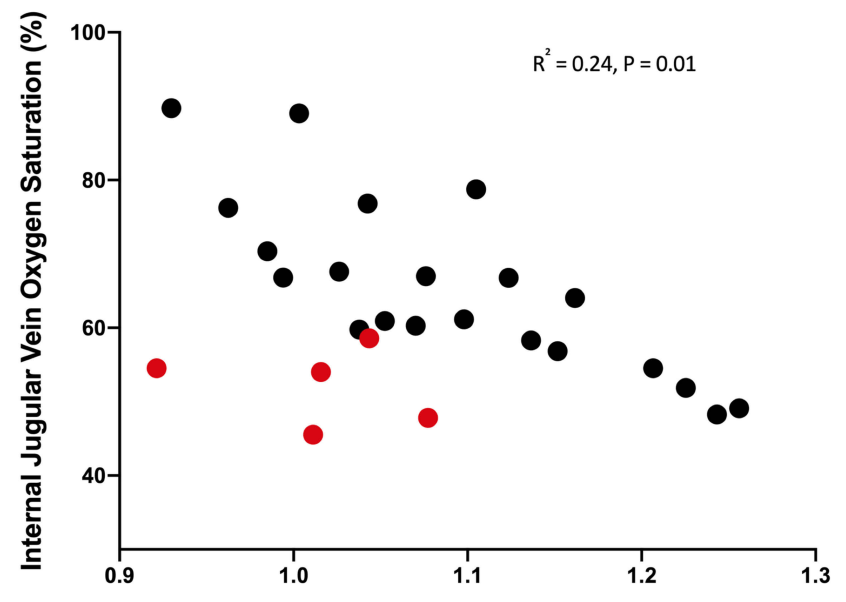

Brain oximeter Modified Ratio of Ratio's

Figure 8 Correlation between oxygen saturation measured from proximal internal jugular vein blood gas samples and the modified ratio of ratios of the brain oximeter. $R^{2} 0.24, p=0.01, R=-0.49(95 \% \mathrm{Cl}-0.74$ to -0.13$)$. The data is from 3 subjects. The red circles represent values from one volunteer in which the brain PPG waveform demonstrated an earlier and steeper rise during the systolic phase in PPG waveform. We speculate this brain oximeter PPG waveform may represent periods of very high cerebral perfusion.

characteristics of the PPG waveform and large changes in oxygen saturations during the cardiac cycle suggest the brain oximeter signal reflected (predominately) blood volume and oxygen levels in the venular component of the brain microcirculation. The systolic increase in oxygen levels may represent arterial blood entering the microcirculation, while the diastolic fall in oxygen may represent oxygen diffusing out into the tissues. By contrast, skin oxygen levels remained at arterial levels throughout systole and diastole, presumably due to shunting through the skin, bypassing capillary beds. ${ }^{26}$

\section{Responses of Brain and Skin Oximeter Oxygen Saturations and PPG Waveforms to Hypoxia}

The brain and skin oximeter responses to hypoxia were significantly different. The extent of fall, from baseline, in brain oxygen saturations was $1 / 3$ that of the skin. At the point of maximum hypoxia, skin oxygen saturation (averaged over systole and diastole) fell $37 \%$, from $87 \%$ to $50 \%$, while brain oximeter oxygen saturation fell only $12 \%$, from $86 \%$ to $74 \%$. This finding is consistent with the expected regional blood flow responses to acute hypoxia. In animal studies, acute hypoxia increases cerebral blood flow, which limits the fall in brain microvascular oxygen levels, while skin blood flow does not increase. $^{15,16}$ Human trials, using MRI, doppler techniques or measurement of jugular bulb venous oxygen saturations, also demonstrate cerebral blood flow increases by $\sim 20 \%$ to limit the fall in brain oxygen levels. ${ }^{27-32}$

\section{Responses in Brain and Skin Oxygen Saturations with Change from $10 \%$ to 100\% Inspired Oxygen}

The temporal changes in brain oximeter and skin oxygen levels were distinct following the increase in inspired oxygen from $\sim 10-100 \%$. Brain oximeter oxygen saturations rapidly 
increased over 5 seconds to hyperoxic levels (99\%) and fell thereafter, while skin oxygen levels increased slowly over 25 seconds. These distinct responses may represent the tight auto-regulation of cerebral blood flow in comparison to that of the skin, to maintain stable brain oxygen levels. ${ }^{33}$ The acute increase in inspired oxygen may have resulted in a rapid over-shoot of brain oxygen saturation, due to the concurrent high cerebral blood flow. Cerebral autoregulation with vasoconstriction may then have resulted in a fall in cerebral blood flow to normalise brain oxygen saturations. These distinct temporal responses in oxygen levels in the brain and skin suggest that the brain oximeter was not markedly contaminated by skin oxygen levels, as is a limitation of existing cerebral oximeters. ${ }^{18}$

During periods of hypoxia, the brain PPG waveform developed an earlier and steeper rise during the systolic phase compared with the baseline PPG waveform, and the $\mathrm{V}$ and $\mathrm{Y}$ waves were less prominent. These changes may represent increased cerebral blood flow, resulting in an earlier increase of blood volume in the microcirculation of the brain and therefore an earlier upstroke in the PPG waveform. These changes were not observed in the simultaneous skin PPG waveform.

\section{Oscillations}

Episodes of high-frequency oscillations, at around $7 \mathrm{~Hz}$, developed in the brain oximeter PPG waveform following the transitions to lower inspired oxygen levels. These oscillations were not present in the skin PPG waveform. The synchronous nature of the oscillations with the pulse was consistent with a cardiac source. Oscillations at this frequency have also been noted in the pulsations of intracranial pressure (ICP) catheter waveforms and may represent mechanical movement or "ringing of the brain" in response to the systolic blood pressure wave entering the brain. ${ }^{34-36}$ Bench-based models that reproduced the effect of the systolic blood pressure wave on the brain found the brain oscillated at $\sim 10 \mathrm{~Hz}$, with an amplitude of $\sim 10 \mathrm{mmHg} .{ }^{37}$ Acute hypoxia, to the levels produced in this study, triggers a $\sim 20 \%$ increase in cerebral blood flow. ${ }^{29-31}$ Increased cerebral blood flow could increase the pulsatile mechanical forces acting on the brain, and thus the amplitude of the brain oscillations, to a level detectable by the brain oximeter.

Recent improvements in MRI imaging with cardiac gating have enabled movements of the brain due to the systolic blood pressure wave to be documented. The movements were found to be most marked in the central brain structures. ${ }^{38,39}$ See https://directorsblog.nih.gov/2018/06/21/brain-in-motion/.
The mechanisms resulting in high-altitude cerebral oedema (HACE) remain undefined. ${ }^{27}$ Our finding that high-frequency oscillations developed in the brain during periods of acute hypoxia raises the possibility that additional mechanical forces acting on the brain, through increased cerebral blood flow, could over the hours or days while climbers are exposed to hypoxia, cause physical injury to the brain. MRI studies of patients with HACE demonstrated brain injury typically involved the central brain structures, supporting this hypothesis. $^{40,41}$

The EEG, a conventional approach to brain monitoring, also detects oscillations, in the $7 \mathrm{~Hz}$ range, in the normal brain. These oscillations are believed to arise from intrinsic neural activity; however, a number of studies have demonstrated associations between cerebral blood flow or heart rate and the amplitude of these oscillations, suggesting that brain movement due to the mechanical forces associated with the systolic pressure wave is an alternative mechanism. ${ }^{37,42-46}$

\section{Correlation}

We found a relatively weak correlation between the brain oximeter's $R R_{m}$ and the internal jugular vein (IJV) blood oxygen saturations. One important limitation of our methodology was that the IJV blood was taken from the proximal end of the jugular vein, and not the jugular bulb. This approach was used as this was a feasibility study that assessed oxygen levels, at a number of internal sites, in addition to the brain. Blood in the proximal IJV also includes blood that drains from extracranial sites. This diminishes the strength of the relationship of proximal IJV oxygen levels with cerebral oxygen levels. ${ }^{47}$ The brain oximeter measured the average oxygen saturations over the entire cardiac cycle. As the systolic phase represents arterial oxygen levels this could weaken the relationship with venous oxygen saturations. Finally, the correlation was strengthened by the exclusion of values from one subject in which the brain oximeter waveform demonstrated an earlier and steeper rise during the systolic phase of the PPG waveform. We have speculated this PPG waveform may represent a marked increase in cerebral blood flow, in which case, brain oxygen levels may be well preserved relative to extra-cranial tissues and, if so, contribute to a poorer correlation with proximal IJV blood oxygen levels.

\section{Limitations}

The relationship between the $R_{m}$ derived from the brain optical signal and brain oxygen saturations is as yet been established. We therefore used the known relationship 
between RR and blood oxygen saturations, of conventional skin pulse oximetry to estimate oxygen saturations of blood in the brain. Furthermore, with very low oxygen saturations, less than $75 \%$, the correlation between RR and blood oxygen levels weakens, adding greater uncertainty to these estimates. ${ }^{48}$ This preliminary approach can only provide a guide to the relative changes in brain oxygen levels. Six volunteers were studied but oxygen data were only available in 3. Further, larger studies are therefore required to confirm these preliminary results.

\section{Conclusion}

Brain oximeter oxygen saturations were relatively well preserved compared to the skin during acute hypoxia. These findings are consistent with the expected physiological responses and suggest skin oxygen levels did not markedly contaminate the brain oximeter signal.

\section{Abbreviations}

ECG, electrocardiography; $\mathrm{F}_{\mathrm{i}} \mathrm{O}_{2}$, inspired concentration of oxygen fraction; IJV, internal jugular vein; ICP, intracranial pressure; $\mathrm{RR}_{\mathrm{m}}$, modified Ratio of Ratios; NIR, near infra-red; $\mathrm{SO}_{2}$, oxygen saturation; $\mathrm{RR}$, Ratio of Ratios; TBI, traumatic brain injury.

\section{Data Sharing Statement}

The datasets used and/or analysed during the current study are available from the corresponding author on reasonable request.

\section{Author Contributions}

All authors made substantial contributions to conception and design, acquisition of data, or analysis and interpretation of data; took part in drafting the article or revising it critically for important intellectual content; gave final approval of the version to be published; and agree to be accountable for all aspects of the work.

\section{Funding}

This research received no specific grant from any funding agency in the public or not-for-profit sectors and was funded by Cyban Pty Ltd.

\section{Disclosure}

Dr Dixon has a financial interest in Cyban Pty Ltd, that is developing the brain oximeter and has a patent brain oximeter pending. The Human Pharmacology and Physiology Laboratory, Department of Anesthesiology, and School of
Nursing, Duke University, Durham, NC received fees from Cyban Pty Ltd, for conduct of the study. The authors report no other conflicts of interest in this work.

\section{References}

1. Maloney-Wilensky E, Gracias V, Itkin A, et al. Brain tissue oxygen and outcome after severe traumatic brain injury: a systematic review. Crit Care Med. 2009;37(6):2057-2063. doi:10.1097/CCM.0b013e3181a009f8

2. Oddo M, Levine JM, Mackenzie L, et al. Brain hypoxia is associated with short-term outcome after severe traumatic brain injury independently of intracranial hypertension and low cerebral perfusion pressure. Neurosurgery. 2011;69(5):1037-1045. doi:10.1227/NEU. 0b013e3182287ca7

3. Stiefel MF, Spiotta A, Gracias VH, et al. Reduced mortality rate in patients with severe traumatic brain injury treated with brain tissue oxygen monitoring. J Neurosurg. 2005;103(5):805-811. doi:10.3171/ jns.2005.103.5.0805

4. Okonkwo DO, Shutter LA, Moore C, et al. Brain oxygen optimization in severe traumatic brain injury phase-II: a phase II randomized trial. Crit Care Med. 2017;45(11):1907-1914. doi:10.1097/CCM.000 0000000002619

5. Xie Q, Wu HB, Yan YF, Liu M, Wang ES. Mortality and outcome comparison between brain tissue oxygen combined with intracranial pressure/cerebral perfusion pressure-guided therapy and intracranial pressure/cerebral perfusion pressure-guided therapy in traumatic brain injury: a meta-analysis. World Neurosurg. 2017;100:118-127. doi:10.1016/j.wneu.2016.12.097

6. Barone DG, Czosnyka M. Brain monitoring: do we need a hole? An update on invasive and noninvasive brain monitoring modalities. Sci World J. 2014;2014:795762. doi:10.1155/2014/795762

7. Llompart-Pou JA, Barea-Mendoza JA, Sanchez-Casado M, et al. Neuromonitoring in the severe traumatic brain injury. Spanish trauma ICU registry (RETRAUCI). Neurocirugia (Astur). 2019.

8. Cnossen MC, Huijben JA, van der Jagt M, et al. Variation in monitoring and treatment policies for intracranial hypertension in traumatic brain injury: a survey in 66 neurotrauma centers participating in the CENTER-TBI study. Crit Care. 2017;21(1):233. doi:10.1186/ s13054-017-1816-9

9. Leal-Noval SR, Cayuela A, Arellano-Orden V, et al. Invasive and noninvasive assessment of cerebral oxygenation in patients with severe traumatic brain injury. Intensive Care Med. 2010;36 (8):1309-1317. doi:10.1007/s00134-010-1920-7

10. Caccioppola A, Carbonara M, Macri M, et al. Ultrasound-tagged near-infrared spectroscopy does not disclose absent cerebral circulation in brain-dead adults. $B r J$ Anaesth. 2018;121(3):588-594. doi:10.1016/j.bja.2018.04.038

11. Schneider A, Minnich B, Hofstatter E, Weisser C, HattingerJurgenssen E, Wald M. Comparison of four near-infrared spectroscopy devices shows that they are only suitable for monitoring cerebral oxygenation trends in preterm infants. Acta Paediatr. 2014;103(9):934-938. doi:10.1111/apa.12698

12. Steppan J, Hogue CW Jr. Cerebral and tissue oximetry. Best Pract Res Clin Anaesthesiol. 2014;28(4):429-439. doi:10.1016/j.bpa. 2014.09.002

13. Lund A, Secher NH, Hirasawa A, et al. Ultrasound tagged near infrared spectroscopy does not detect hyperventilation-induced reduction in cerebral blood flow. Scand J Clin Lab Invest. 2016;76 (1):82-87. doi:10.3109/00365513.2015.1101485

14. Dixon B, Turner R, Christou C. Assessment of a non-invasive brain oximeter in a sheep model of acute brain injury. Med Devices (Auckl). 2019;12:479-487. doi:10.2147/MDER.S235804

15. Adachi H, Strauss W, Ochi H, Wagner HN Jr. The effect of hypoxia on the regional distribution of cardiac output in the dog. Circ Res. 1976;39(3):314-319. doi:10.1161/01.RES.39.3.314 
16. Todd MM, Wu B, Maktabi M, Hindman BJ, Warner DS. Cerebral blood flow and oxygen delivery during hypoxemia and hemodilution: role of arterial oxygen content. Am J Physiol. 1994;267(5 Pt 2): H2025-2031. doi:10.1152/ajpheart.1994.267.5.H2025

17. Kato S, Yoshitani K, Kubota Y, Inatomi Y, Ohnishi Y. Effect of posture and extracranial contamination on results of cerebral oximetry by near-infrared spectroscopy. J Anesth. 2017;31(1):103-110. doi:10.1007/s00540-016-2275-1

18. Davie SN, Grocott HP. Impact of extracranial contamination on regional cerebral oxygen saturation: a comparison of three cerebral oximetry technologies. Anesthesiology. 2012;116(4):834-840. doi:10.1097/ALN.0b013e31824c00d7

19. Mendelson Y. Pulse oximetry: theory and applications for noninvasive monitoring. Clin Chem. 1992;38(9):1601-1607. doi:10.1093/ clinchem/38.9.1601

20. Garcia-Lopez I, Rodriguez-Villegas E. Extracting the jugular venous pulse from anterior neck contact photoplethysmography. Sci Rep. 2020;10(1):3466. doi:10.1038/s41598-020-60317-7

21. Hirsch S, Reichold J, Schneider M, Szekely G, Weber B. Topology and hemodynamics of the cortical cerebrovascular system. J Cereb Blood Flow Metab. 2012;32(6):952-967. doi:10.1038/jcbfm.2012.39

22. Lee SP, Duong TQ, Yang G, Iadecola C, Kim SG. Relative changes of cerebral arterial and venous blood volumes during increased cerebral blood flow: implications for BOLD fMRI. Magn Reson Med. 2001;45(5):791-800. doi:10.1002/mrm.1107

23. Wiedeman MP. Dimensions of blood vessels from distributing artery to collecting vein. Circ Res. 1963;12(4):375-378. doi:10.1161/01. RES.12.4.375

24. Santisakultarm TP, Cornelius NR, Nishimura N, et al. In vivo two-photon excited fluorescence microscopy reveals cardiac- and respiration-dependent pulsatile blood flow in cortical blood vessels in mice. Am J Physiol Heart Circ Physiol. 2012;302(7):H1367-1377. doi:10.1152/ajpheart.00417.2011

25. Zweifach BW. Quantitative studies of microcirculatory structure and function. II. Direct measurement of capillary pressure in splanchnic mesenteric vessels. Circ Res. 1974;34(6):858-866. doi:10.1161/01. RES.34.6.858

26. Walloe L. Arterio-venous anastomoses in the human skin and their role in temperature control. Temperature (Austin). 2016;3(1):92-103. doi:10.1080/23328940.2015.1088502

27. Sagoo RS, Hutchinson CE, Wright A, et al. Magnetic resonance investigation into the mechanisms involved in the development of high-altitude cerebral edema. J Cereb Blood Flow Metab. 2017;37 (1):319-331. doi:10.1177/0271678X15625350

28. Wilson MH, Edsell ME, Davagnanam I, et al. Cerebral artery dilatation maintains cerebral oxygenation at extreme altitude and in acute hypoxia-an ultrasound and MRI study. J Cereb Blood Flow Metab. 2011;31(10):2019-2029. doi:10.1038/jcbfm.2011.81

29. Harris AD, Murphy K, Diaz CM, et al. Cerebral blood flow response to acute hypoxic hypoxia. NMR Biomed. 2013;26(12):1844-1852 doi:10.1002/nbm.3026

30. Iwasaki K, Zhang R, Zuckerman JH, Ogawa Y, Hansen LH, Levine BD. Impaired dynamic cerebral autoregulation at extreme high altitude even after acclimatization. J Cereb Blood Flow Metab. 2011;31(1):283-292. doi:10.1038/jcbfm.2010.88

31. Arngrim N, Hougaard A, Schytz HW, et al. Effect of hypoxia on BOLD fMRI response and total cerebral blood flow in migraine with aura patients. J Cereb Blood Flow Metab. 2019;39(4):680-689. doi:10.1177/0271678X17719430
32. Schober A, Feiner JR, Bickler PE, Rollins MD. Effects of changes in arterial carbon dioxide and oxygen partial pressures on cerebral oximeter performance. Anesthesiology. 2018;128(1):97-108. doi:10.1097/ALN.0000000000001898

33. Haggendal E, Johansson B. Effects of arterial carbon dioxide tension and oxygen saturation on cerebral blood flow autoregulation in dogs. Acta Physiol Scand Suppl. 1965;258:27-53. doi:10.1111/j.17481716.1965.tb03234.x

34. Lang EW, Paulat K, Witte C, Zolondz J, Mehdorn HM. Noninvasive intracranial compliance monitoring. Technical note and clinical results. J Neurosurg. 2003;98(1):214-218. doi:10.3171/jns.2003.98.1.0214

35. Wagshul ME, Eide PK, Madsen JR. The pulsating brain: a review of experimental and clinical studies of intracranial pulsatility. Fluids Barriers CNS. 2011;8(1):5. doi:10.1186/2045-8118-8-5

36. Robertson CS, Narayan RK, Contant CF, et al. Clinical experience with a continuous monitor of intracranial compliance. J Neurosurg. 1989;71(5 Pt 1):673-680. doi:10.3171/jns.1989.71.5.0673

37. Castillo HT. A cardiac hypothesis for the origin of EEG alpha. IEEE Trans Biomed Eng. 1983;30(12):793-796. doi:10.1109/TBME.1983. 325080

38. Holdsworth SJ, Rahimi MS, Ni WW, Zaharchuk G, Moseley ME. Amplified magnetic resonance imaging (aMRI). Magn Reson Med. 2016;75(6):2245-2254. doi:10.1002/mrm.26142

39. Greitz D, Wirestam R, Franck A, Nordell B, Thomsen C, Stahlberg F. Pulsatile brain movement and associated hydrodynamics studied by magnetic resonance phase imaging. The Monro-Kellie doctrine revisited. Neuroradiology. 1992;34(5):370-380. doi:10.1007/BF00596493

40. Hackett PH, Yarnell PR, Hill R, Reynard K, Heit J, McCormick J. High-altitude cerebral edema evaluated with magnetic resonance imaging: clinical correlation and pathophysiology. JAMA. 1998;280 (22):1920-1925. doi:10.1001/jama.280.22.1920

41. Medhi G, Lachungpa T, Saini J. Neuroimaging features of fatal high-altitude cerebral edema. Indian J Radiol Imaging. 2018;28 (4):401-405. doi:10.4103/ijri.IJRI 29618

42. Mullinger KJ, Havenhand J, Bowtell R. Identifying the sources of the pulse artefact in EEG recordings made inside an MR scanner. Neuroimage. 2013;71:75-83. doi:10.1016/j.neuroimage.2012.12.070

43. O'Gorman RL, Poil -S-S, Brandeis D, et al. Coupling between resting cerebral perfusion and EEG. Brain Topography. 2013;26(3):442-457. doi:10.1007/s10548-012-0265-7

44. Chang LJ, Lin JF, Lin CF, Wu KT, Wang YM, Kuo CD. Effect of body position on bilateral EEG alterations and their relationship with autonomic nervous modulation in normal subjects. Neurosci Lett. 2011;490(2):96-100. doi:10.1016/j.neulet.2010.12.034

45. Guger C, Domej W, Lindner G, Pfurtscheller K, Pfurtscheller G, Edlinger G. Effects of a fast cable car ascent to an altitude of 2700 meters on EEG and ECG. Neurosci Lett. 2005;377(1):53-58. doi:10.1016/j.neulet.2004.11.065

46. Ozaki H, Watanabe S, Suzuki H. Topographic EEG changes due to hypobaric hypoxia at simulated high altitude. Electroencephalogr Clin Neurophysiol. 1995;94(5):349-356. doi:10.1016/0013-4694(94) 00311-8

47. Chieregato A, Calzolari F, Trasforini G, Targa L, Latronico N. Normal jugular bulb oxygen saturation. J Neurol Neurosurg Psychiatry. 2003;74(6):784-786. doi:10.1136/jnnp.74.6.784

48. Carter BG, Carlin JB, Tibballs J, Mead H, Hochmann M, Osborne A. Accuracy of two pulse oximeters at low arterial hemoglobin-oxygen saturation. Crit Care Med. 1998;26(6):1128-1133. doi:10.1097/ 00003246-199806000-00040 


\section{Publish your work in this journal}

Medical Devices: Evidence and Research is an international, peerreviewed, open access journal that focuses on the evidence, technology, research, and expert opinion supporting the use and application of medical devices in the diagnosis, monitoring, treatment and management of clinical conditions and physiological processes. The identification of novel devices and optimal use of existing devices

which will lead to improved clinical outcomes and more effective patient management and safety is a key feature of the journal The manuscript management system is completely online and includes a very quick and fair peer-review system. Visit http:// www.dovepress.com/testimonials.php to read real quotes from published authors. 